\title{
Investigation of Noise-Resolution Tradeoff for Digital Radiographic Imaging: A Simulation Study
}

\author{
Eri Matsuyama1, Du-Yih Tsai ${ }^{1}$, Yongbum Lee ${ }^{1}$, Katsuyuki Kojima ${ }^{2}$ \\ ${ }^{1}$ Department of Radiological Technology, Graduate School of Health Sciences, Niigata University, Niigata, Japan; ${ }^{2}$ Department of \\ Business Administration, Graduate School of Business Administration, Hamamatsu University, Hamamatsu, Japan. \\ Email: meri@cyber.ocn.ne.jp, \{tsai, lee\}@clg.niigata-u.ac.jp, kojima@hamamatsu-u.ac.jp
}

Received July 16 ${ }^{\text {th }}$ 2010; revised August $9^{\text {th }}, 2010$; accepted August $16^{\text {th }}, 2010$.

\begin{abstract}
In digital radiographic systems, a tradeoff exists between image resolution (or blur) and noise characteristics. An imaging system may only be superior in one image quality characteristic while being inferior to another in the other characteristic. In this work, a computer simulation model is presented that is to use mutual-information (MI) metric to examine tradeoff behavior between resolution and noise. MI is used to express the amount of information that an output image contains about an input object. The basic idea is that when the amount of the uncertainty associated with an object before and after imaging is reduced, the difference of the uncertainty is equal to the value of MI. The more the MI value provides, the better the image quality is. The simulation model calculated MI as a function of signal-to-noise ratio and that of resolution for two image contrast levels. Our simulation results demonstrated that MI associated with overall image quality is much more sensitive to noise compared to blur, although tradeoff relationship between noise and blur exists. However, we found that overall image quality is primarily determined by image blur at very low noise levels.
\end{abstract}

Keywords: Modeling and Simulation, Medical Imaging, Image Quality Evaluation, Mutual Information

\section{Introduction}

The modulation transfer function (MTF), noise power spectrum (NPS), and detective quantum efficiency are commonly used as image quality metrics to characterize resolution, noise, and efficiency performance of digital radiographic systems, respectively [1-3]. These metrics are dealt with in the spatial frequency domain. Recently, an information-entropy based approach has been reported for evaluating overall image quality in medical imaging systems [4-6]. In these reports, transmitted information (TI) $[4,5]$ or mutual information (MI) [6] was used as an image quality criterion. Both TI and MI were defined as "the amount of shared information", i.e., "the amount of information transmitted from stimulus (input) to response (output)". The more the transmitted information provides, the better the image quality is. Therefore, the overall quality of an image can be quantitatively evaluated by measuring TI (or MI). Unlike the physical performance measures, the information entropy-based metric is dealt with in the spatial domain.

One of the current dilemmas in digital radiography is the extent to which these parameters such as, resolution and noise affect physical or clinical image quality. An imaging system may only be superior in one metric while being inferior to another in the other metric. In general, higher spatial resolution leads to an increased noise level. Simulation studies of image quality attributes for x-ray systems using computer methods have been performed by several investigators, and shown to be effective methods of evaluating various elements of the image formation process $[7,8]$. A computer simulation approach was also presented to investigate the impact of image quality metrics on the appearance of radiographic images [9]. The approach was to emulate the influence of resolution and noise characteristics of a digital detector on the appearance of a radiographic image. Recently, attention has been paid to address the tradeoff between spatial resolution and quantum noise relation for computed tomography and digital radiography [10-12]. In these studies, it is of general nature that the MTF and NPS were used as the descriptors of spatial resolution and noise. However, we believe that it is also interesting to attempt to understand the tradeoff in terms of image information 
such as mutual information.

In this paper, a computer simulation approach is presented that is to employ the MI metric to investigate tradeoff behavior between resolution and noise. The simulation model calculated $\mathrm{MI}$ as a function of signalto-noise ratio and that of resolution for two specific image contrast levels. Two simulation studies were per- formed separately; the first simulation was carried out to investigate the relationship between image blurring and MI for various levels of noise, and the second simulation was conducted to investigate the relationship between image noise and MI for different extent of blurring. In this work, a total of 2,688 simulations were performed in order to conduct a detailed analysis and achieve a better understanding of noise-resolution tradeoff.

\section{Theoretical Framework}

MI is a concept from information theory $[13,14]$ and is also referred to as TI as described in the section of preceding Section [4-6]. MI has been applied in medical image processing, particularly for image registration [15-18]. The definition of the term of MI has been presented in various ways in the literature. We will briefly describe MI, as used in the image evaluation sense, rather than as used in the image registration sense.

Given events $s_{1}, \ldots \ldots s_{n}$ occurring with probabilities $p_{1}$, $p_{2}, \ldots \ldots p_{\mathrm{n}}$, the Shannon entropy $H$ is defined as

$$
H\left(p_{1}, p_{2}, \ldots p_{n}\right)=-\sum_{i=1}^{n} p_{i} \log _{2} p_{i}
$$

Considering $x$ and $y$ as two random variables corresponding to an input variable and an output variable, the entropy for the input and that for the output are denoted as $H(x)$ and $H(y)$, respectively. For this case, the MI can be defined as

$$
\begin{aligned}
M I(x ; y) & =H(x)-H_{y}(x)=H(y)-H_{x}(y) \\
& =H(x)+H(y)-H(x, y)
\end{aligned}
$$

where $H(x, y)$ is the joint entropy, and $H_{x}(y)$ and $H_{y}(x)$ are conditional entropies. The entropies and joint entropy are given as

$$
\begin{gathered}
H(x)=-\sum_{i} p_{i} \log _{2} p_{i} \\
H(y)=-\sum_{j} p_{j} \log _{2} p_{j} \\
H(x, y)=-\sum_{i j} p_{i j} \log _{2} p_{i j}
\end{gathered}
$$

where $p_{i}$ and $p_{j}$ are the marginal probabilities, and $p_{i j}$ is the joint probability.

A useful way of visualizing the relationship among these entropies is provided by a Venn diagram as shown in Figure 1. The MI measures how much the uncertainty of input $x$ is known if output $y$ has been given. It can be easily shown that if input and output are generally independent, then $H(x, y)=H(x)+H(y)$. Consequently, their $\mathrm{MI}$ is zero (i.e., transmitted information is equal to zero). In other words, observing $y$ does not reduce the uncertainty of $x$. If, however, $x=y$, i.e., $H(x)=H(y)$, then $\mathrm{MI}=$ $H(x)$. Under this condition, the information about input $x$ can be obtained completely. We apply the MI measure to evaluate image quality of digital radiography based on the following reasoning. Consider an experiment in which every input has a unique output belonging to one of the various output categories. The inputs may be considered to be a set of subjects, for example, a test sample object with steps of various thickness, whereas the outputs may be their corresponding images varying in optical density or gray level. If the inputs can be recognized completely when the outputs have shown, then the quality or the performance of the transmission channel of the system (i.e., imaging system), can be perfect. In the cur- rent study, a method of occurrence-frequency-based computation was used for calculating the entropies of input, output, and their joint entropies. The details of the calculation procedure can be found in the literature [4-6].

\section{Methods}

\subsection{Computer Simulation}

A simulation was designed, and its framework is as follows. A simulation image $g(x, y)$ was given by a spatial convolution between a uniformly-distributed signal (an object) $f(x, y)$ having intrinsic noise $u(x, y)$ and a blurring function $B$. If the external noise $v(x, y)$ was also taken into consideration, the resulting image could be represented by the following formula:

$$
\begin{aligned}
g(x, y)= & \sum_{s=1}^{5}\{[s \times f(x, y)+u(x, y) \times W] * B \\
& +v(x, y) \times K\},
\end{aligned}
$$

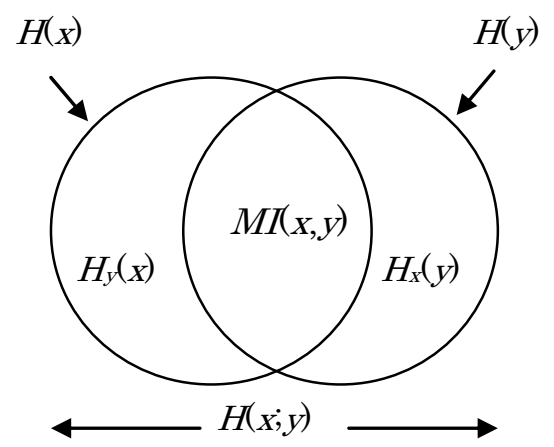

Figure 1. The relations between conditional and joint entropies, and the mutual information. 
where the symbol $*$ represents the convolution operation, and $s$ is an integer representing the number of steps of the simulated image. The terms of $W$ and $K$ are weighting coefficients used to adjust noise level.

In the simulation studies, the input image $f(x, y)$ was a five-step wedge with a specific intensity or a pixel value on each step. Both $u(x, y)$ and $v(x, y)$ were zero-mean Gaussian noise with a standard deviation of 0.5 . In the studies, for simplicity, the term of $v(x, y) \times K$ was considered the external noise and is equal to the intrinsic noise of $u(x, y) \times W$ (i.e., $u(x, y) \times W=v(x, y) \times K)$. We used a " $m \times m$ " ( $m$ is an odd integer) 8-neighborhood averaging filter as the blurring function. The extent of blurring was adjusted by varying the filter size (FS). The reason for choosing neighborhood averaging filter was its ease of implementation and effectiveness, which were confirmed by experiments.

Two simulations were performed. The first simulation was carried out to investigate the relationship between image blur and MI for various noise levels at specific image contrasts levels. In this study, we defined image contrast as the difference of the mean pixel values between two adjacent steps of a simulated step wedge. We used signal-to-noise ratio (SNR) to describe the extent of noise level. Notice that the signal and noise used for SNR calculation were $f(x, y)$ and $u(x, y) \times W$, respectively, as given in Equation (6). In this work, combinations of 64 various SNRs (range, 24-43 dB), 21 various FSs (range, 3-41), and two different contrast levels (20 and 40) were used for simulation studies. As a result, a total of 2688 simulations were performed for the analysis of resolution-noise tradeoff.

The second simulation was performed to investigate the relationship between image noise and MI for different extent of blurring at specific image contrast levels.

\subsection{Step-wedge Phantom Images}

For verification and validation of our designed computer simulation models, phantom images of an acrylic step-wedge with $2,4,6,8$, and $10 \mathrm{~mm}$ in thickness were obtained using the following exposure conditions. The specific exposure factors were kept at $42 \mathrm{kV}$ and $10 \mathrm{~mA}$, the focus-imaging distance was taken at $185 \mathrm{~cm}$, and the exposure time was varied ranging from 0.1 to $0.5 \mathrm{sec}$. An imaging plate for computed radiography was used as a detector to record $\mathrm{x}$-ray intensities.

\section{Simulation Results and Discussion}

Figures $\mathbf{2}$ and $\mathbf{3}$ compare the computer-simulated images versus the phantom images obtained. The simulated images shown in the figures were generated using Equation (6). A perceptual comparison of the simulated images and phantom images indicates that these images were

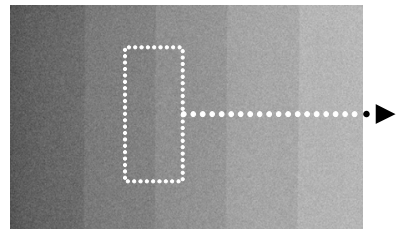

(a)

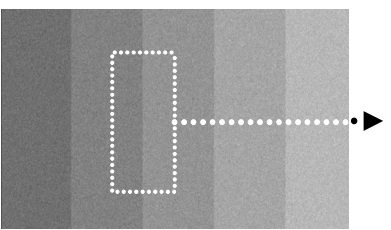

(d)

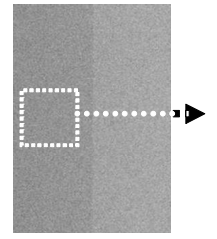

(b)

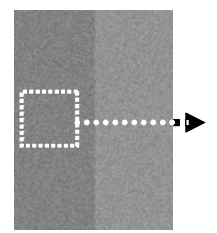

(e)

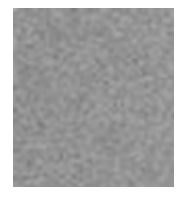

(c)

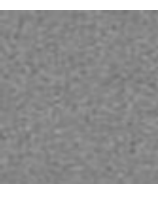

(f)
Figure 2. Perceptual evaluation of computer simulated images. (a) Computer-simulated image. The parameters used are: $W=130, \mathrm{SNR}=26.1 \mathrm{~dB}$, contrast $=70, \mathrm{FS}=3$. (b) The magnified image from the rectangular area shown in (a). (c) The magnified image from the rectangular area shown in (b). (d) Step wedge phantom image. Exposure time was $0.5 \mathrm{~s}$. The step wedge was placed $30 \mathrm{~cm}$ apart from the center toward the cathode end for imaging. (e) The magnified image from the rectangular area shown in (d). (f) The magnified image from the rectangular area shown in (e).

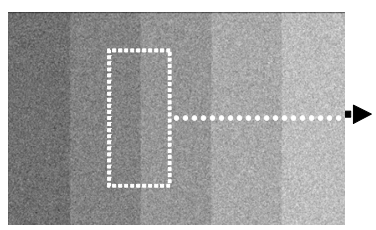

(a)

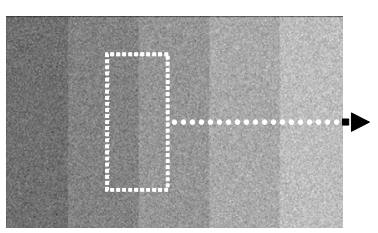

(d)

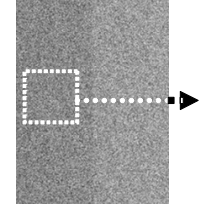

(b)

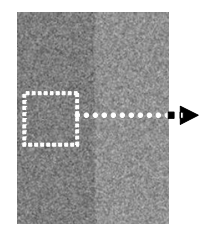

(e)

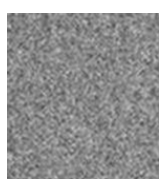

(c)

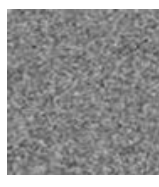

(f)
Figure 3. Perceptual evaluation of computer simulated images. (a) Computer-simulated image. The parameters used are: $W=300, \mathrm{SNR}=19.3 \mathrm{~dB}$, contrast $=70, \mathrm{FS}=3$. (b) The magnified image from the rectangular area shown in (a). (c) The magnified image from the rectangular area shown in (b). (d) Step wedge phantom image. Exposure time was 0.1 s. The step wedge was placed $30 \mathrm{~cm}$ apart from the center toward the cathode end for imaging. (e) The magnified image from the rectangular area shown in (d). (f) The magnified image from the rectangular area shown in (e).

very similar in appearance with respect to noise, blur and visibility of detail. The comparison result indicated that our designed mathematical model provides a good means of simulating the resolution and noise characteristics of digital radiographic systems. 
Figure 4 illustrates MI as a function of FS for varying levels of SNR ranging from 24 to $41 \mathrm{~dB}$ at an image contrast of 40. It should be noted that FS is associated with the extent of blurring: the greater the FS value is, the higher the extent of blurring becomes. As shown in Figure 4, MI decreases with the increase of FS (i.e., increase of image blur) when noise levels are very low (i.e., high SNR; for example, SNR $>36 \mathrm{~dB}$ in this study), although the decrease is relative small. This means that, in the case of low noise levels, the effect of the level of blur on the $\mathrm{MI}$ is not so obvious in comparison to noise.

When noise increases to medium levels (for example, $36 \mathrm{~dB} \geq \mathrm{SNR} \geq 31 \mathrm{~dB}$ in this report), MI initially increases with the increase of FS and then gradually decreases after reaching the maximum value. The increase in MI value might be because that, in spite of deterioration of image resolution, the increase of FS could give rise to a significant decrease of noise. Thus, MI is greatly dependent on the decrease of noise level compared to the deterioration of image resolution. However, on the contrary, when FS increases to a certain level, the MI value is greatly influenced by the deterioration of resolution as compared to that by the decrease of noise.

In the case of high noise levels (for example, SNR $\leq$ $28 \mathrm{~dB}$ ), MI increases gradually with the increase of FS until reaching its maximum value. After that, MI value showed insignificant decreasing. The reasoning could be made as follows: 1) initially, the increase of FS might result in a great decrease of noise, and this yields the increase of MI, although the increase of FS itself might give rise to a small decrease of MI. In other words, the decrease of noise level dominated the variation of MI. 2) However, when FS continued increasing, a tradeoff point appeared. The location, indicated by an arrow on each graph shown in Figure 4, was referred to as the tradeoff point in this study. The location corresponds to the maximum value of MI. For instance, the tradeoff points for $\mathrm{SNR}=41,31$, and $25 \mathrm{~dB}$ can be found at $\mathrm{FS}=3,9$, and 17 , respectively. It is noted that, not surprisingly, the location of the tradeoff point depends on SNR. An oblique line in the figure depicts the trend in the change of the location. As shown in the figure, MI reaches to its maximum at a finer resolution when SNR increases.

Figure 5 plots MI as a function of FS for varying levels of SNR at an image contrast of 20. Overall, the trend of this case is similar to that at image contrast of 40. It can be seen from Figures $\mathbf{4}$ and $\mathbf{5}$ that images with higher contrast show greater MI values in comparison to those with lower contrast, if both images have the same spatial resolution (same FS) and the same noise level (same SNR). It is reasonable to conclude that a higher contrast image shows better image quality.

Figure 6 shows MI as a function of SNR for various

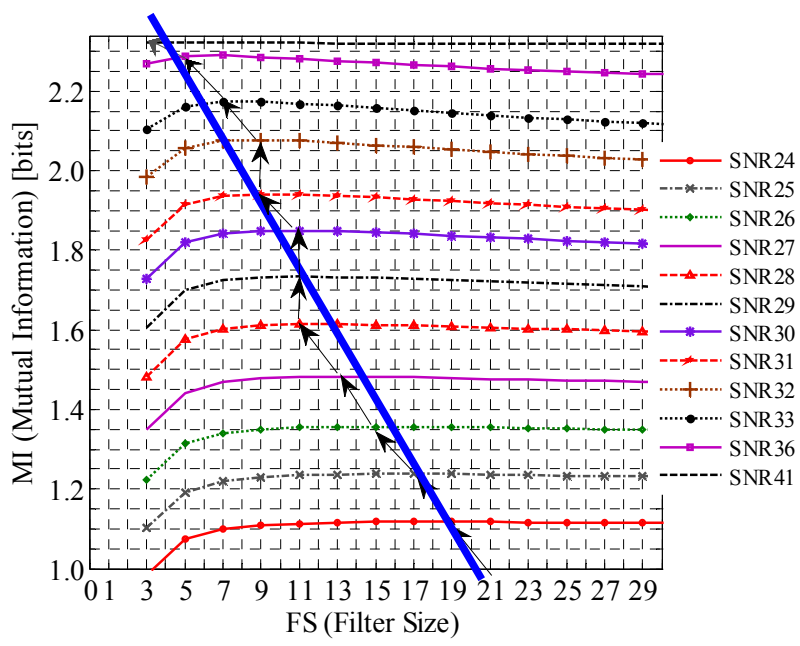

Figure 4. Relationship between FS and MI for varying levels of SNR at an image contrast of 40 .

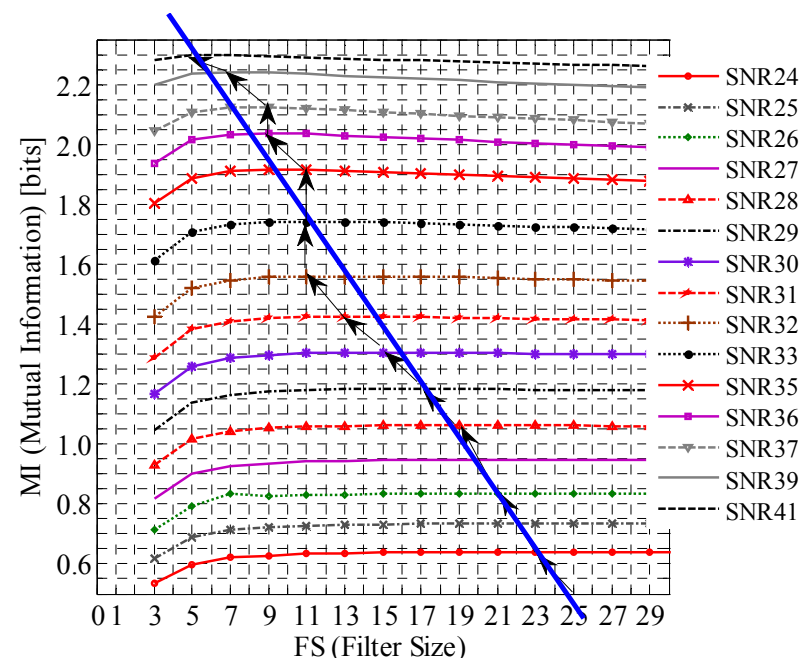

Figure 5. Relationship between FS and MI for varying levels of SNR at an image contrast of 20.

sizes of FS at an image contrast of 40 . The results indicate that, basically, MI value increases with the increase of SNR (decrease in noise level). The figure illustrates only the results covering a range of SNR from 29 to $36 \mathrm{~dB}$, where intersections between the line graphs occur. The intersection between two graphs indicates that two images have the same overall image quality, although the images may show different extent of blur. For instance, the intersection between the graphs of FS $=3$ and $\mathrm{FS}=41$ is located near SNR $=33$. It is noted that an image blurred by a smoothing filter of FS $=41$ might decrease the MI value because of resolution deterioration caused by blurring. On the contrary, the MI value might increase because of the decrease of noise resulting from smoothing operation. This means that the physical quality of an image is mutually adjusted by resolution 
and noise properties at a specific image contrast. The result implies that the contribution of resolution attribute and that of noise attribute to the MI value vary depending on the levels of noise and blur. Here, it should be noted that the external noise (i.e., the term of $v(x, y) \times K$ shown in equation (6)) also served as a factor that influences overall image quality of an image.

Figure 7 shows MI as a function of SNR for various sizes of FS at image contrast of 20. The figure also shows that the MI value increases with the increase of SNR. From Figures 6 and 7, it is noted that the MI value for the image with lower contrast is lower than that with higher contrast. However, the trends of the two cases are similar. As described earlier, a higher contrast image shows better image quality compared to a lower contrast image.

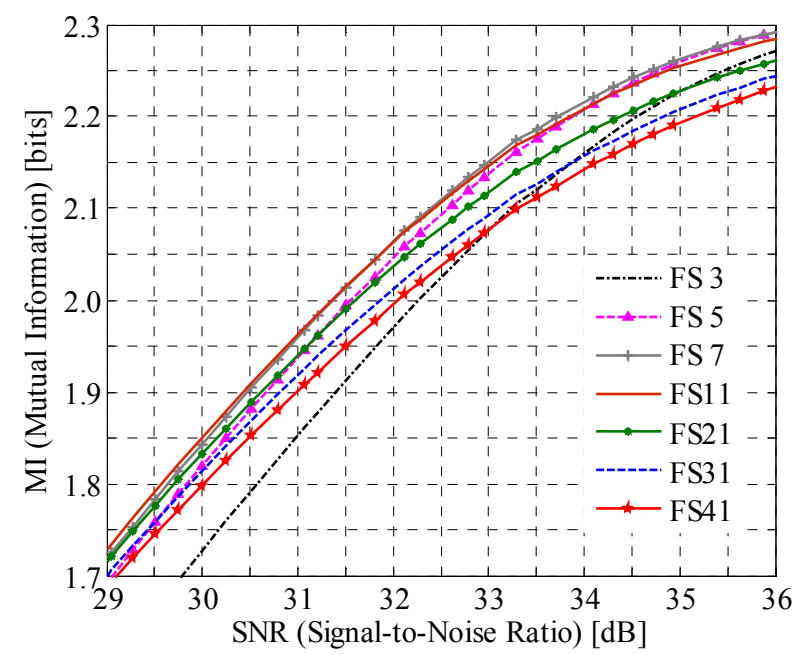

Figure 6. Relationship between SNR and MI for varying sizes of $\mathrm{FS}$ at an image contrast of $\mathbf{4 0 .}$

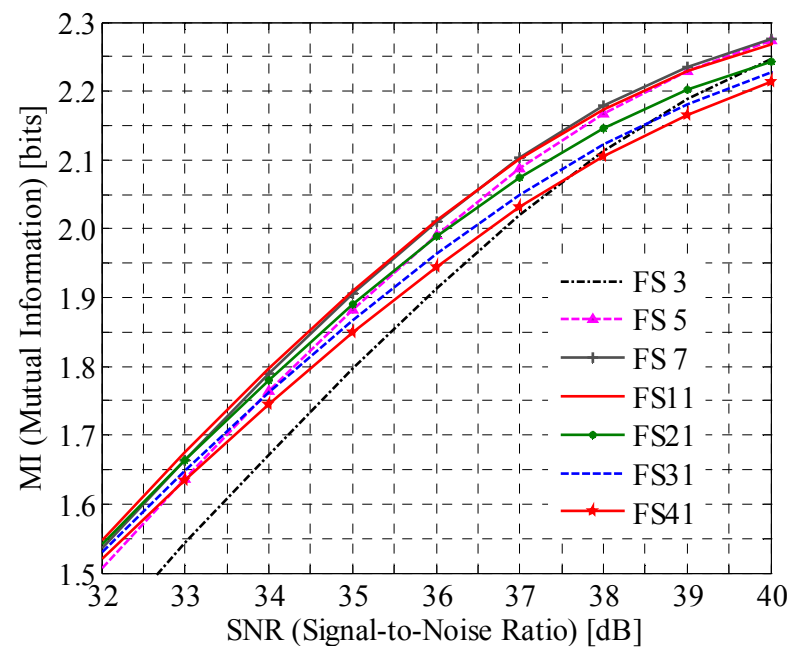

Figure 7. Relationship between SNR and MI for varying sizes of FS at an image contrast of 20 .
Figures 8 and 9 show the MI values, plotted as a function of SNR and FS (blur) at image contrast of 40 and 20, respectively. Figure 8 corresponds to Figures $\mathbf{4}$ and $\mathbf{6}$, while Figure $\mathbf{9}$ corresponds to Figures 5 and 7 . As shown in the figures, MI reaches to the maximum value when an image has very high SNR. Moreover, it is noted that $\mathrm{MI}$ is much more sensitive to noise compared to blur. It is also noted that the image with a higher contrast level provides greater MI and shows better image quality in comparison with that with lower contrast level, if the two images have the same noise and blurring levels (Figures 8 and 9).

Figures $\mathbf{1 0}$ and $\mathbf{1 1}$ are plots of SNR (noise) versus FS (blur) for four different MI values (i.e., 2.276, 2.206, 2.159 , and 2.090), corresponding to four various transmitted efficiency (i.e., 98\%, 95\%, 93\%, and 90\%) for the

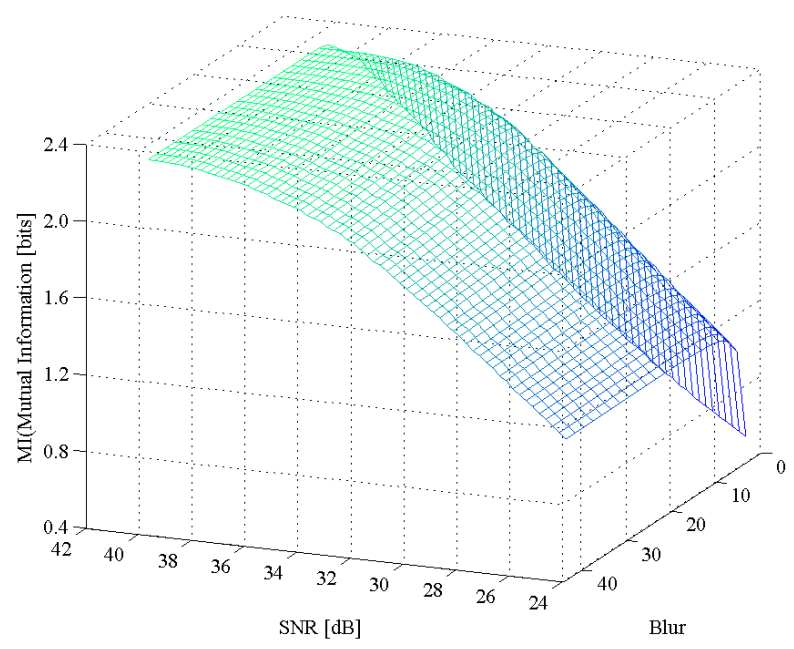

Figure 8. MI calculated as a function of SNR and FS (blur) at image contrast of 40 .

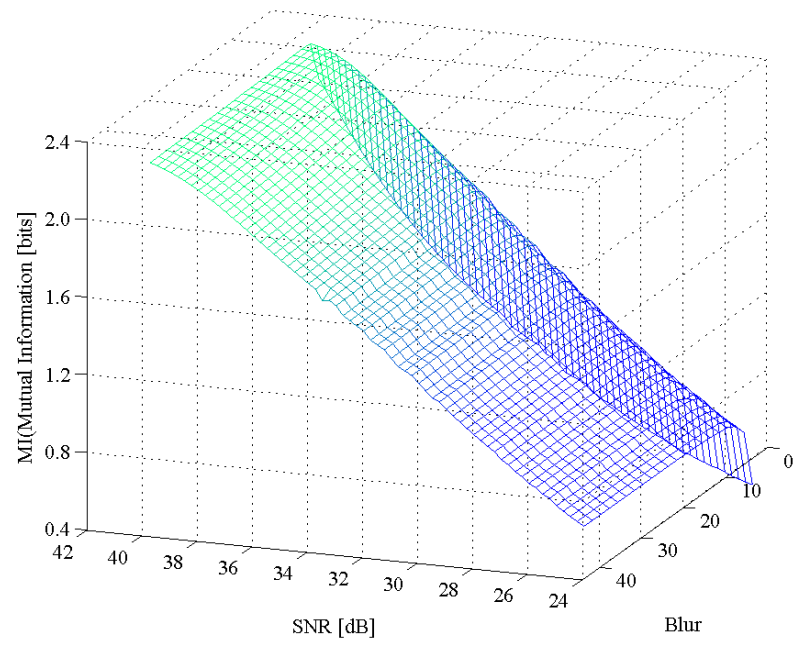

Figure 9. MI calculated as a function of SNR and FS (blur) at image contrast of 20 . 


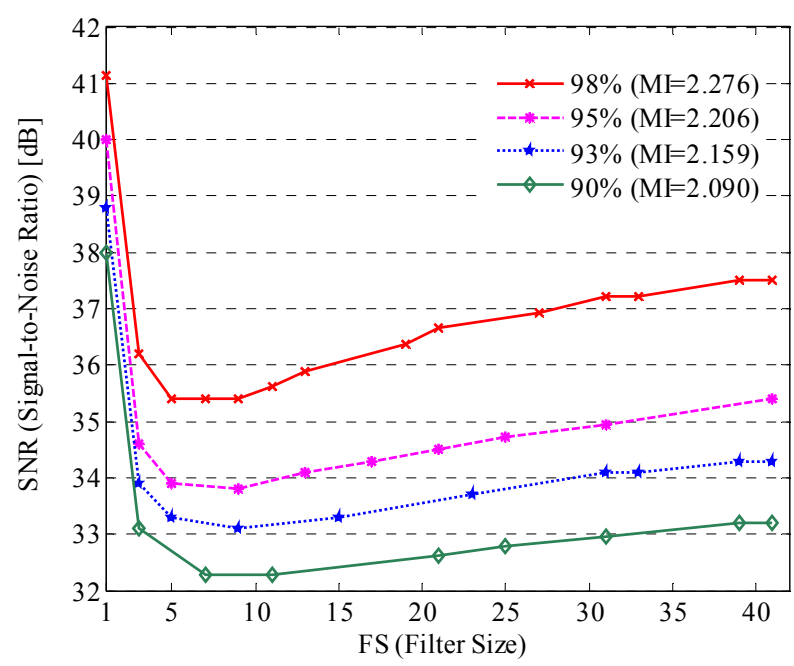

Figure 10. A plot of SNR versus FS for four different MI values at an image contrast of 40 .

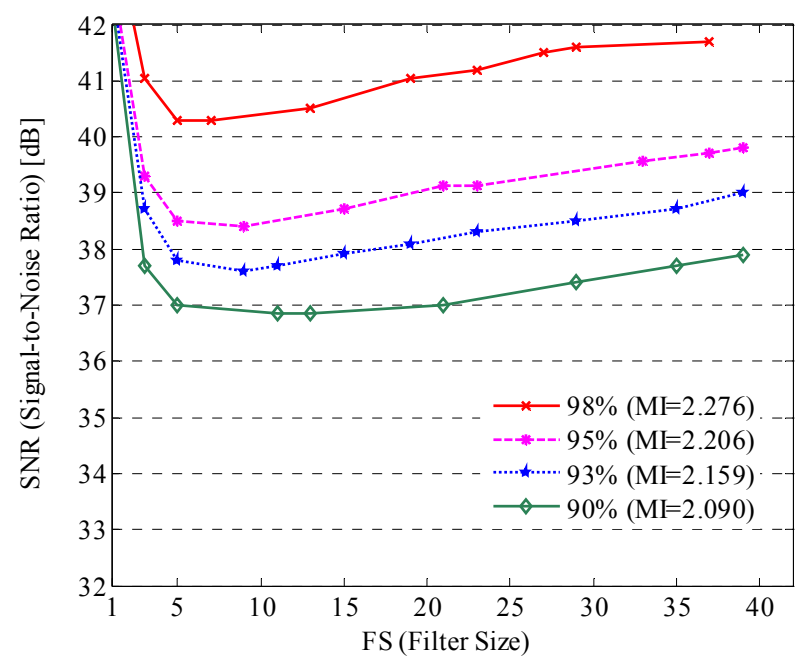

Figure 11. A plot of SNR versus FS for four different MI values at an image contrast of 20 .

cases of contrast levels of 40 and 20, respectively. In this study, "transmission efficiency" was used and defined as the ratio between $\mathrm{MI}$ and the input entropy, i.e., $\eta=(\mathrm{MI} / H(x)) \%$. The efficiency $\eta$ may also be used as a measure for indicating how good the imaging quality of an image receptor is. Points shown on each curve in the figures, obtained from different combinations of SNR and FS, have the same MI values, thus providing the same overall image quality. It is observed that a minimum point exists at each graph. Noted that only high SNR levels (i.e., low noise level) ranging from 32 to 42 $\mathrm{dB}$ are depicted in Figures 10 and 11. Because the minimum points for those SNR levels lower than $32 \mathrm{~dB}$ did not appear in our simulation studies. As shown in the two figures, tradeoff relationship between image noise and blur exists on the right of the minimum point. This means that a combination of a lower noise level and a deteriorated resolution might provide the same physical image quality as a combination of a higher noise level and a higher resolution level. On the left of the minimum point, however, image quality is primarily determined by resolution when the SNR of an image is higher than 32 $\mathrm{dB}$ in our investigation. In other words, the image quality of a very-low-noise image is almost determined by the extent of blur, even when noise level had slight variations. There might be two reasons for this. First, for images of very low noise levels, image quality might not be affected by small change of noise levels. As described in the section of Theoretical Framework, MI measures how much the uncertainty of input is known if output has been given. As a result, very small changes in the amount of noise might not influence the amount of the uncertainty. Second, as shown in Figures $\mathbf{4}$ and 5, MI has a significant increase with the increase of deterioration of resolution at low FS values (range, 3-9 in the simulation studies).

It must be addressed here that the purpose of this study was to present a computer simulation approach to investigate tradeoff behavior between resolution and noise using the MI metric. In order to validate the results obtained from this study, we will perform visual evaluation and compare them in the future work.

Our simulation results showed that the proposed simulation approach by employing mutual-information metric to examine tradeoff behavior between resolution and noise is useful, reliable and challenging.

\section{Conclusions}

A computer simulation study for examining resolutionnoise tradeoff behavior has been presented. In this study MI was used as an image quality metric for the analysis. Our simulation results demonstrated that the MI value associated with overall image quality is much more sensitive to noise compared to blur, although tradeoff relation between noise and blur exists. However, at very low noise levels (SNR values higher than $32 \mathrm{~dB}$ ), we found that overall image quality is primarily determined by image blur. However, a comparison between the result of physical evaluation and that of perceptual evaluation was not made in this work. It would be a very interesting research question for our future study.

\section{REFERENCES}

[1] H. Fujita, K. Doi and M. L. Giger, "Investigation of Basic Imaging Properties in Digital Radiography 6 MTFs of II-TV Digital Imaging Systems," Medical Physics, Vol. 12, No. 6, 1985, pp. 713-720. 
[2] M. L. Giger, K. Doi and H. Fujita, "Investigation of Basic Imaging Properties in Digital Radiography 7 Noise Wiener Spectra of II-TV Digital Imaging Systems," Medical Physics, Vol. 13, No. 2, 1986, pp. 131-138.

[3] A. Maidment and M. Yaffe, "Analysis of Spatial-Frequency-Dependent DQE of Optically Coupled Digital Mammography Detectors," Medical Physics, Vol. 21, No. 6, 1994, pp. 721-729.

[4] E. Matsuyama, D. Y. Tsai, Y. Lee, M. Sekiya and K. Kojima, "Physical Characterization of Digital Radiological Images by Use of Transmitted Information Metric," Proceedings of 2008 SPIE Medical Imaging, Vol. 6913, 2008, pp. 69130V1-V8.

[5] D. Y. Tsai, Y. Lee and E. Matsuyama, "Information Entropy Measure for Evaluation of Image Quality," Journal of Digital Imaging, Vol. 21, No. 3, 2008, pp. 338-347.

[6] E. Matsuyama, D. Y. Tsai and Y. Lee, "Mutual Information-Based Evaluation of Image Quality with Its Preliminary Application to Assessment of Medical Imaging Systems," Journal of Electronic Imaging, Vol. 18, No. 3, 2009, pp. 033011-1-11.

[7] R. Nishikawa and S. Lee, "Preliminary Results on a Method for Producing Simulated Mammograms," presented at the AAPM 44th Annual Meeting, Montreal, Quebec, Canada, 2002.

[8] A. Tingberg, C. Herrmann, J. Besjakov, A. Almen, P. Sund, D. Adliene, S. Mattsson, L. Mansson and W. Panzer, "What is Worse: Decreased Spatial Resolution or Increased Noise," Proceedings of SPIE Medical Imaging, Vol. 4686, 2002, pp. 338-346.

[9] R. S. Saunders Jr. and E. Samei, “A Method for Modifying the Image Quality Parameters of Digital Radiographic Images," Medical Physics, Vol. 30, No. 11, 2003, pp. 30063017.

[10] T. Fuchs and W. Kalender, "On the Correlation of Pixel
Noise, Spatial Resolution and Dose in Computed Tomography," Physica Medica, Vol. XIX, No. 2, 2003, pp. 153164.

[11] B. Li, G. B. Avinash and J. Hsieh, "Resolution and Noise Trade-off Analysis for Volumetric CT," Medical Physics, Vol. 34, No. 10, 2007, pp. 3732-3738.

[12] A. R. Pineda and H. H. Barrett, "What Does DQE Say about Lesion Detectability in Digital Radiography," Proceedings of 2001 SPIE Medical Imaging, Vol. 4320, 2001, pp. 561-569.

[13] C. E. Shannon, "Mathematical Theory of Communication," Bell System Technology Journal, Vol. 27, 1948, pp. 379-423, pp. 623-656.

[14] C. E. Shannon and W. Weaver, "The Mathematical Theory of Communication," The University of Illinois Press, Urbana, 1948.

[15] D. Skerl, B. Likar and F. Pernus, "A Protocol for Evaluation of Similarity Measures for Rigid Registration," IEEE Transactions on Medical Imaging, Vol. 25, No. 6, 2006, pp. 779-791.

[16] G. D. Tourassi, B. Harrawood, S. Singh and J. Y. Lo, "Information-Theoretic CAD System in Mammography: Entropy-Based Indexing for Computational Efficiency and Robust Performance," Medical Physics, Vol. 34, No. 8, 2007, pp. 3193-3204.

[17] J. P. W. Pluim, J. B. A. Maintz amd M. A. Viergever, "Mutual-Information-Based Registration of Medical Images: A Survey," IEEE Transactions on Medical Imaging, Vol. 22, No. 8, 2003, pp. 986-1004.

[18] G. D. Tourassi, E. D. Frederick, M. K. Marley and C. E. Floyd Jr., "Application of the Mutual Information Criterion for Feature Selection in Computer-Aided Diagnosis," Medical Physics, Vol. 28, No. 12, 2001, pp. 2394-2402. 